\title{
Designing an introductory FPGA-based embedded system laboratory
}

\author{
Swapna Chintakunta, Raghavendra Rao Kanchi, Ramanjappa Thogata \\ VLSI and Embedded System Laboratory, Department of Physics, Sri Krishnadevaraya University, ANANTAPURAMU, 515003, India \\ Email address: \\ kanchiraghavendrarao@gmail.com (R. R. Kanchi)
}

To cite this article:

Swapna Chintakunta, Raghavendra Rao Kanchi, Ramanjappa Thogata. Designing an Introductory FPGA-Based Embedded System Laboratory. American Journal of Embedded Systems and Applications. Vol. 2, No. 2, 2014, pp. 6-12. doi: 10.11648/j.ajesa.20140202.11

\begin{abstract}
In this paper, we present a series of experiments useful in designing an Embedded System Laboratory. The experiments are built around the Spartan3 XC3S400 manufactured by Xilinx. The necessary code is written in the Very High Speed Integrated Circuit Hardware Description Language (VHDL). Integrated Simulation Environment (ISE) Version 9.1i suite is used for software development which is one of the Electronic Design Automation (EDA) tool offered by the Xilinx Company. It gives a good beginning for students who want to work on Spartan 3 XC3S400.
\end{abstract}

Keywords: FPGA, Spartan 3, VHDL, Embedded System Laboratory

\section{Introduction}

The impact and presence of embedded system is felt directly in our daily walk of life. Starting with cellular phones, digital cameras, home appliances, space applications up to ubiquitous networking and sensor networking embedded system are used [1]. Traditionally, the designers have three options to realize device hardware control platform. These are Digital Signal Processors (DSPs), Field Programmable Gate Arrays (FPGAs) and Application Specific Integrated Circuits (ASICs). Digital Signal Processors execute only one operation at a time, so the overall algorithm proceeds in a sequential manner. For cost reduction purposes, ASICs are introduced in high volume production series. However, a lot of effort and costs are related with production of specific ASIC, which makes this technology unsuitable for Research and Development (R\&D) stage of a new product. Between these two extremes, FPGAs provide a compromise between DSPs and ASICs, combining many advantages of both. This makes FPGAs suitable in several applications.

FPGA-based devices are especially well suited for building application - specific systems as a starting point in an undergraduate embedded system design course. FPGAbased design have a much shorter design cycle, lower cost and a smoother learning curve than the traditional system-on-chip technology [2]. When it comes to laboratory, the kind of exposure and training the student gets is meager. In certain cases demo experiments were introduced. This hinders the practical training the student is expected to get in the embedded system training laboratory.

Keeping these facts in view point, we have designed and developed certain experiments using the Spartan XC3S400 manufactured by Xilinx [3]. These experiments are presented in this paper. It Starts with LED BLINKY experiment and goes up to the sensor interfacing.

\section{Description to FPGA and Spartan 3}

\subsection{Field Programmable Gate Array (FPGA)}

A field-programmable gate array (FPGA) is an integrated circuit designed to be configured by a customer or a designer after manufacturing in the field and hence called "field-programmable". Field Programmable Gate Array (FPGA) is a general-purpose, multi-level programmable logic device that is customized in the package by the end users. FPGAs are composed of blocks of logic connected with programmable interconnect. The programmable interconnect between blocks allows users to implement multi-level logic, removing many of the size limitations of the Programmable Logic Devices (PLD)-derived two-level logic structure. This extensible architecture can currently support thousands of gates of logical system and speeds in the tens of megahertz.

The size, structure and number of blocks; and the amount and connectivity of interconnect vary considerably among FPGA architectures. This difference in architectures is driven by different programming technologies and different 
target applications of the parts. The system design based on FPGA is flexible with the advantages of parallelism, low cost and low power consumption [4]. FPGAs fall into three groups: Island-style and cellular SRAM-programmed devices; channeled, antifuse-programmed devices; and array-style EPROM or EEPROM-programmed devices. The target FPGA device used in the present work is XC3S400 SPQ208 of Spartan 3 family. Design, development and debugging are carried on a low cost, full featured kit provided by Applied Digital Microsystems (ADM) [5], connected to desktop computer via JTAG.

\subsection{Spartan 3 Family Packaging Information}

The Spartan 3 family of FPGA is specifically designed to meet the needs of high volume, cost effective consumer electronic applications [6]. Spartan 3 family packaging information is shown in figure 1 .

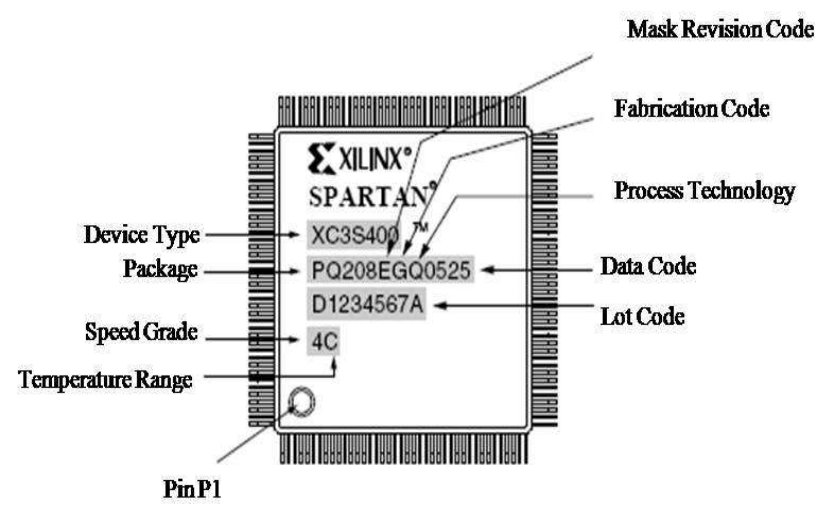

Fig. 1. Spartan 3 Family packaging information

\subsection{Specifications of FPGA Base Board}

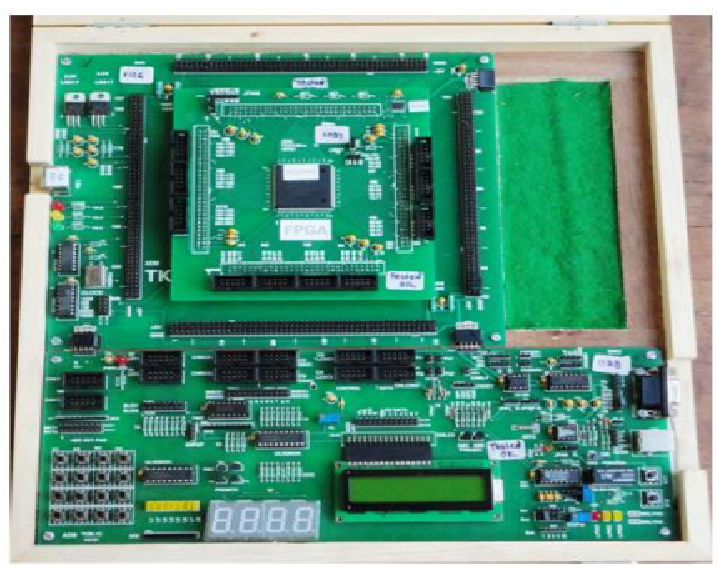

Fig. 2. Photograph of the FPGA base board

The ADM base board is a powerful tool that allows user to understand the capabilities of FPGA. This board acts as a FPGA reconfigurable implementation platform to allow rapid and interactive prototype development. The board houses a plug-in daughter board that contains a target FPGA device. Daughter board can be easily swapped to provide support for multi-vendor devices available in DIL, PLCC,
TQFP, PQFP packages up to 240 pins. Figure 2 shows the photograph of the FPGA base board used in the present work.

The Specifications of base board are as follows [7]

1. $16 \times 2$ Alpha-numeric LCD display with the backlight

2. Four digit 7-segment display, Eight general purpose LEDS

3. Three LEDs to indicate the various power voltages

4. $4 \times 4$ Matrix Keypad

5. $208 \mathrm{I} / \mathrm{O}$ pins, All I/O with VCC/GND links

6. On board $10 \mathrm{MHz}$ oscillators, $10 \mathrm{MHz}$ clock and four different frequency clocks $(5 \mathrm{MHz}, 1 \mathrm{MHz}$, $500 \mathrm{KHz}, 100 \mathrm{KHz})$

7. JTAG Programming Cable, Parallel/Serial/USB/SPI/CAN

8. PS2 Mouse \&Keyboard I/F

9. I2C EEPROM \& RTC, Serial ADC/DAC with Potentiometer

10. RS232 serial interface through 9-PIN D type connector

11. Four sets of $30 \times 2$ male berg connectors to plug the Daughter Board

\subsection{Details of Spartan 3 Daughter Board}

In the present work, daughter board contains Spartan 3 XC3S400 from Xilinx, which can be plugged on to the base board. The Spartan 3 FPGA uses SRAM technology. SRAM Technology is the Static RAM FPGA. Programmable connections are made using pass transistors, transmission gates or multiplexers that are controlled by SRAM cells. The advantage of this technology is that it allows fast in-circuit reconfiguration.

Spartan-3 devices support serial configurations, using the master/slave serial and JTAG modes, as well as byte-wide configuration employing the slave parallel mode. Figure3 shows the photograph of Spartan 3 XC3S400 daughter board used in the present work.

Daughter Board includes the following items

1. Xilinx FPGA Spartan $3 \mathrm{XC} 3 \mathrm{~S} 400$

2. Ten 10-pin Headers, intended for use as GPIO

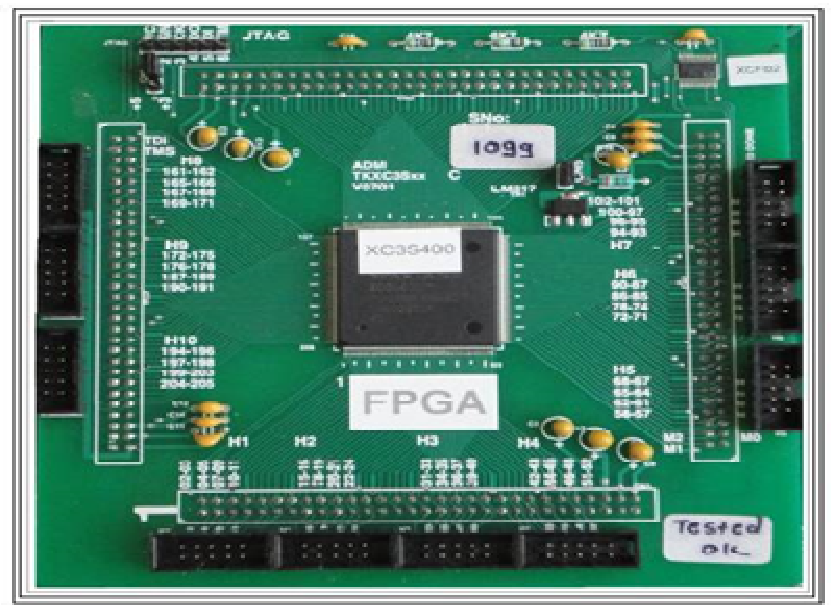

Fig. 3. Photograph of Spartan 3 XC3S400 daughter board 


\section{Details of JTAG Connector}

FPGAs can be configured by using different modes like Boundary Scan, Slave Serial, or Select MAP. The most commonly used mode is Boundary Scan, Also known as JTAG (Joint Test Action Group). The boundary Scan Register and other test features of the device are accessed through a standard interface - the JTAG Test Access Port (TAP). According to this standard, the TAP must contain four signals, TDI (Test Data Input), TDO (Test Data output), TCK (Test Clock), TMS (Test Mode Select) [8]. Parallel download cable is connected to the board through the JTAG port at one end and other end of the cable is connected to PC parallel port.

Xilinx FPGAs are SRAM - based and must be programmed every time power is cycled. The most common method of programming Xilinx FPGAs is by using Xilinx PROM connected to a chain of FPGA as shown in figure 4. These PROMS must be programmed using PROM files created from the FPGA chain bit streams. PROM files include information on the FPGA chain length and contain bit streams that are reformatted for use with PROM file formats available: .mcs,. exo, .rek, hex, .ufp, and. bin. In the present work FPGA is programmed using bit file and PROM is Programmed using .mcs file. Figure 4 shows the JTAG chain configured devices.

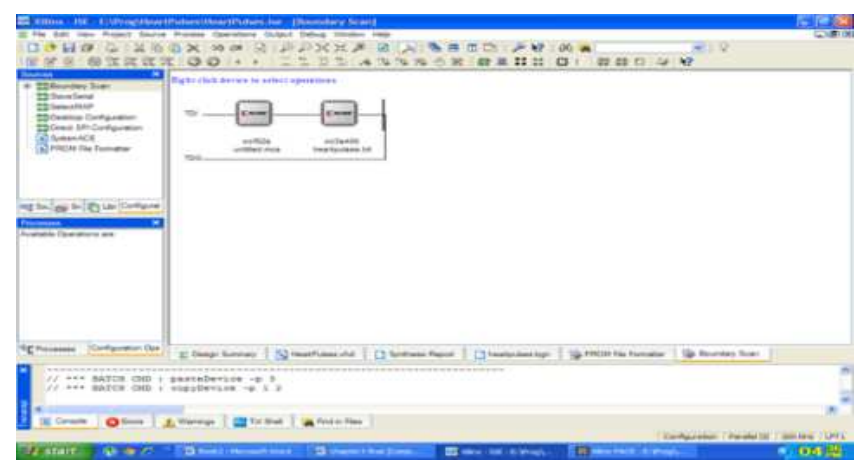

Fig. 4. JTAG Chain the Configured devices

\section{Details of Individual Experiments}

In this paper, the features of Spartan $3 \mathrm{XC} 3 \mathrm{~S} 400$ are used and well explained by the following experiments. These experiments are useful for FPGA beginners. VHDL language is used for programming FPGA. The software tools used for building and testing the experiments in the present work is Xilinx ISE 9.1 i [9].

\subsection{Ex.1.LED Blink Shift}

In this experiment, Header 1 is used as output port. The circuit assembly is shown in figure 5 .

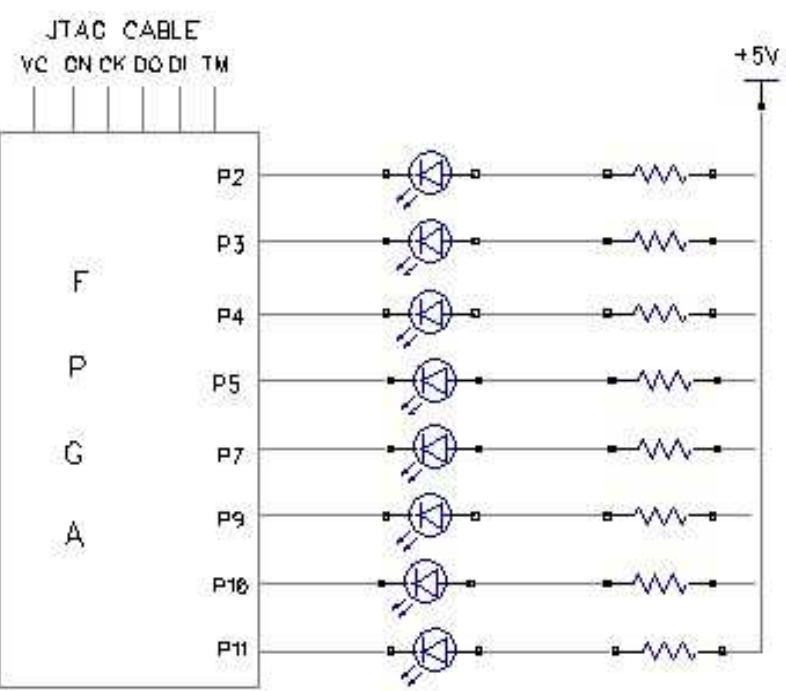

Fig. 5. Schematic diagram of LED Blink Shift

The software is developed basing on the following algorithm.

1. Start.

2. Make Header1 as data out direction.

3. Initialize the header 1 with $0 \times 01$.

4. Realization of led blink shift by making Header1 logical left shift with ' 1 '. This program is kept in continuous loop.

Program describing the above algorithm is written in VHDL language, and the corresponding .mes file is dumped on the flash memory of the Spartan 3 XC3S400. The photograph of the hardware is shown in figure 6 . The blank shift is observed at the bottom of the board.

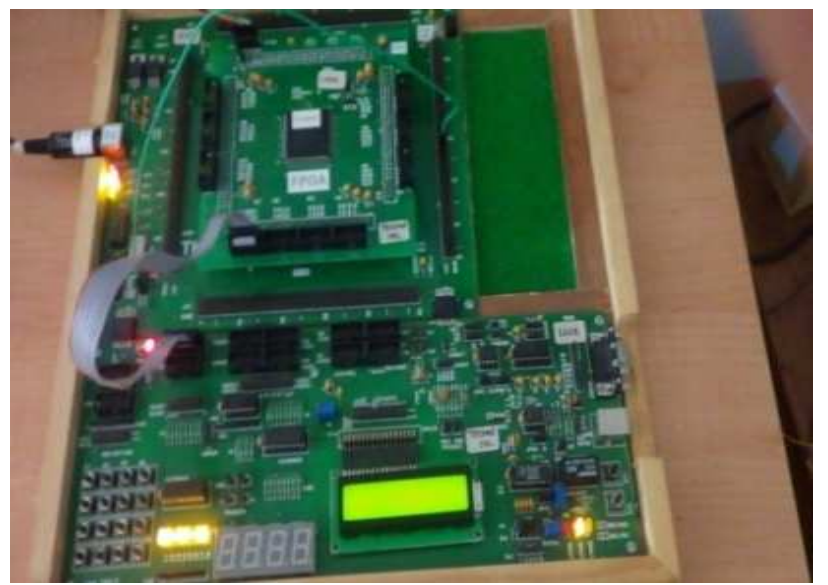

Fig. 6. Photograph of LED blink shift

\subsection{Ex.2.Multiplexed Seven Segment Display Interfacing}

In this experiment Header1 is used for interfacing multiplexed seven segment display and header2 is used to drive the individual segment control signal for individual character. Figure 7 shows the circuit assembly. 


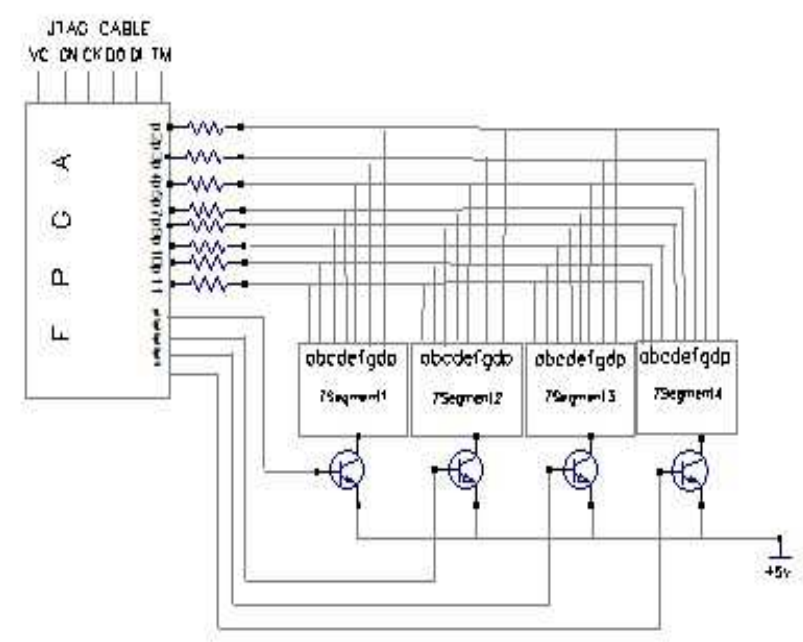

Fig. 7. Schematic diagram of 7-Segment display

The software is developed basing the following algorithm Software is developed basing on the following algorithm.

1. Start

2. Make Header1 as data out direction and Header2 as drive the individual segment control signal for individual segment.

3. Enter the look-up table in the form of array.

4. Keep displaying hex numbers in continuous loop.

The program describing the above algorithm is written in VHDL language and the corresponding .mcs file is dumped on the flash memory of the Spartan 3 XC3S400. Figure 8 shows the photograph of the experiment.

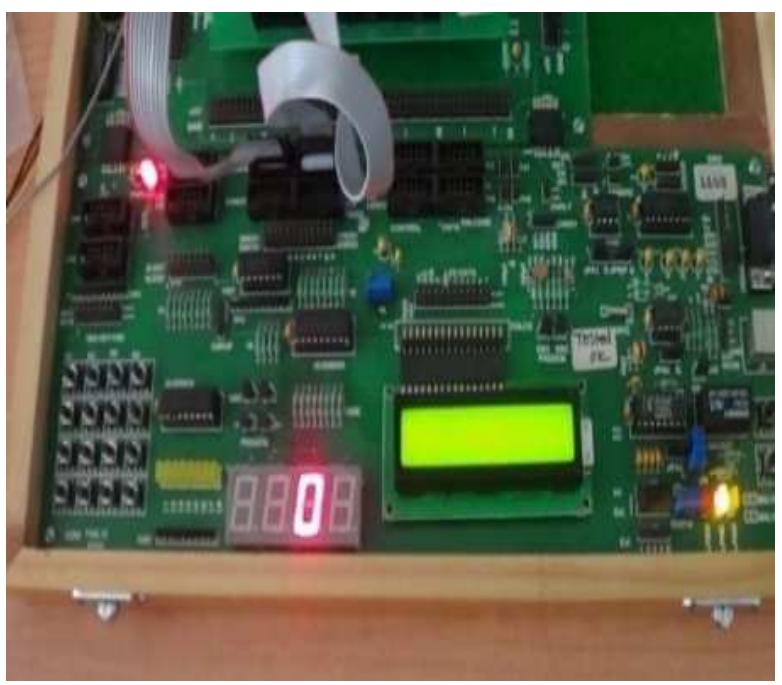

Fig. 8. Photograph of 0-F display on 7-Segment

\subsection{Ex.3.Stepper Motor Interfacing}

In this experiment the stepper motor is interfaced with Spartan 3 XC3S400 using Header1. As the Header1 output of the FPGA cannot drive the stepper motor directly, the power amplifier IC ULN 2003 is used. It provides the necessary current to drive the motor. The circuit assembly is shown in figure 9 .

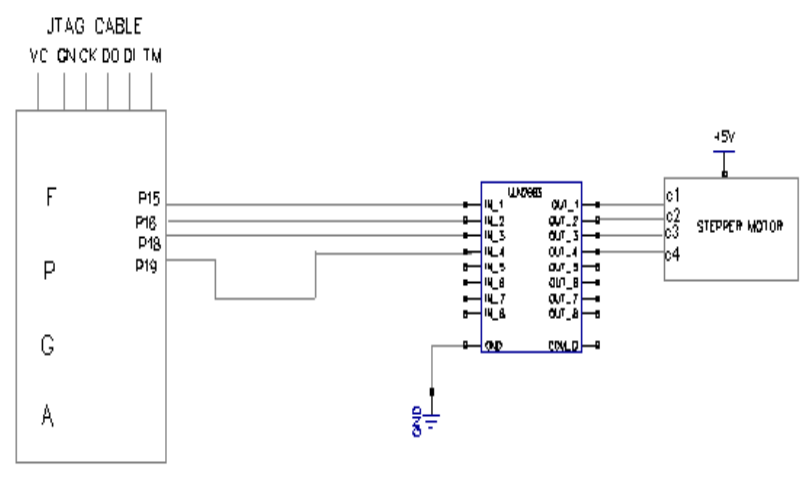

Fig. 9. Schematic diagram of Stepper motor Interface

The stepper motor is rotated in steps continuously, by developing the software basing on the following algorithm.

1. Start.

2. Program higher nibble of Header1 as data out direction.

3. Send the data through Header1.

4. Keep on sending data which rotates motor continuously.

.mcs file developed basing on the above algorithm is dumped on Spartan 3 XC3S400. The figure 10 shows the photograph of the experiment.

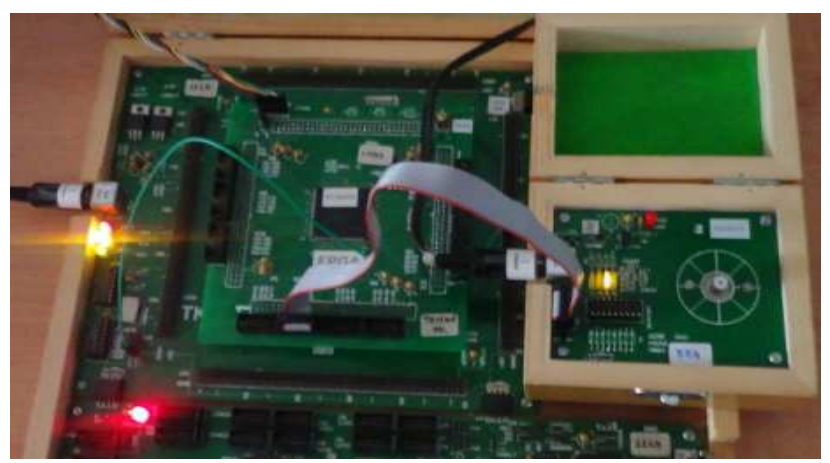

Fig. 10. Photograph of Stepper Motor

\subsection{Ex.4. LCD Interfacing}

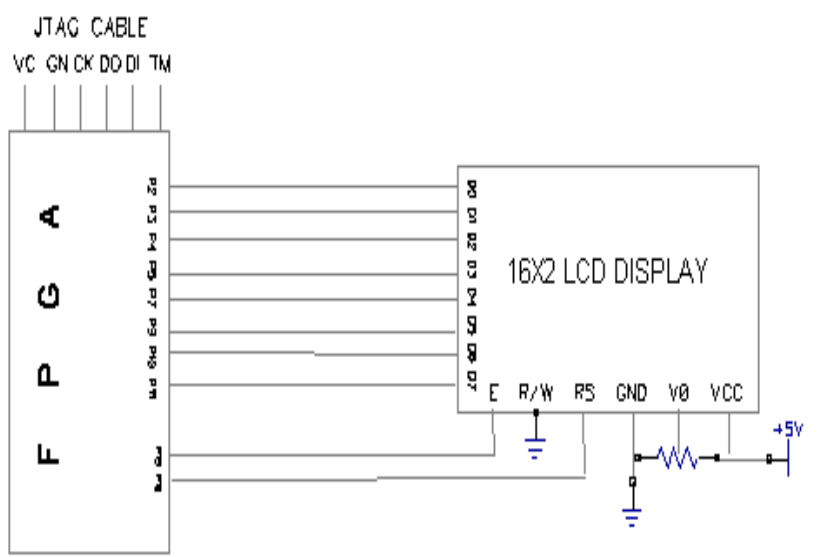

Fig. 11. Schematic diagram of $L C D$ interfacing 
A 16x2 LCD module [10] is used in this experiment; LCD module is used in 8 bit mode. Header1 used for sending data and Header 2 is used as control pins, between FPGA and LCD module. The circuit is assembled as shown in figure11.

Program is developed to display ADM on first and second lines of LCD using the following algorithm:

1. Start

2. Initialize the Header1 and Header2 for LCD communication

3. Initialize LCD

4. Keep on sending characters ADM continuously.

Figure 12 shows the ADM display on LCD module.

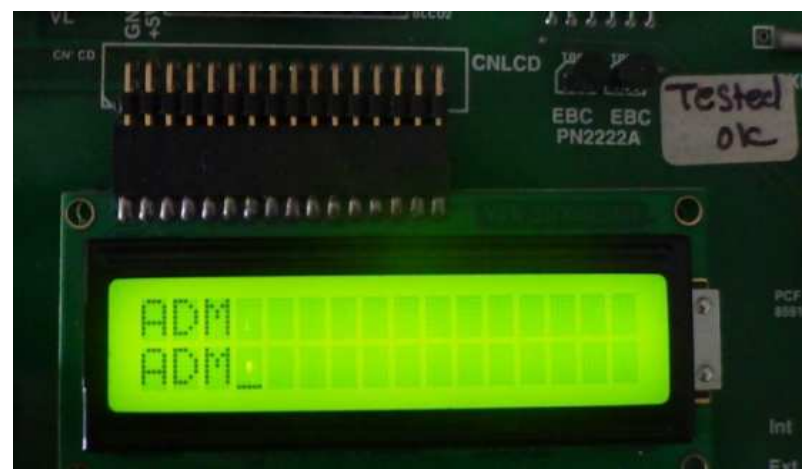

Fig. 12. Photograph of LCD Interfacing

\subsection{Ex.5. ADC 0804 Interfacing}

ADC 0804 is an 8-bit analog-to-digital converter [11] which works on successive approximation principle. In the present setup a $10 \mathrm{k} \Omega / 10$ turn potentiometer is connected to the analog input of ADC 0804. The voltage span is selected as 0 to $5 \mathrm{~V}$. Program is developed to display the digital value corresponding to analog voltage (as read by digital multimeter) is displayed on LCD module. Headers 1 and 2 are programmed as output ports and header 3 as input port. The corresponding circuit is shown is shown in figure 13 .

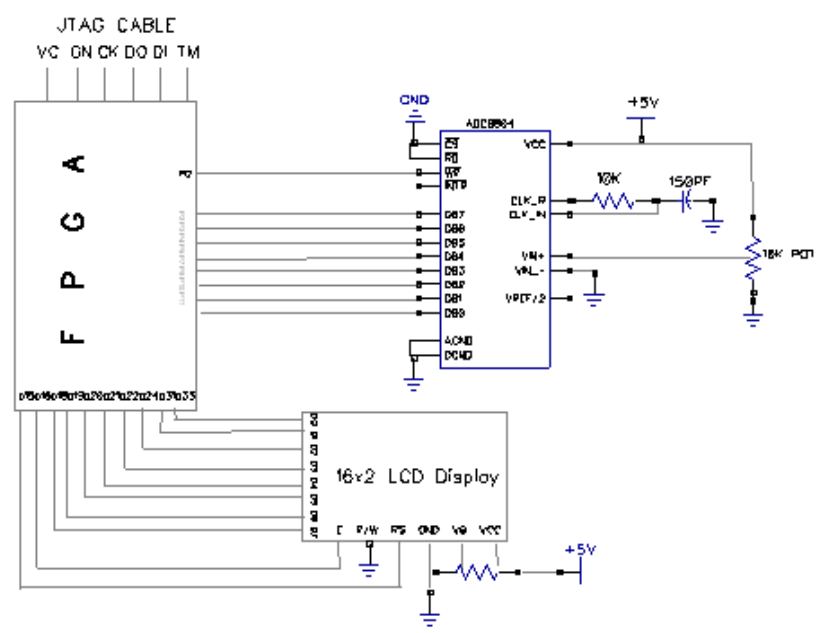

Fig. 13. Schematic diagram of ADC 0804 interfacing

The corresponding algorithm is given below.

1. Start.
2. Initialize the Header1 as output port

3. Initialize the Header2 and Header3 for LCD communication

4. Output data from Header1.

5. Measure the external input voltage and display decimal value on LCD.

The photograph of ADC value display on LCD is shown in figure 14.

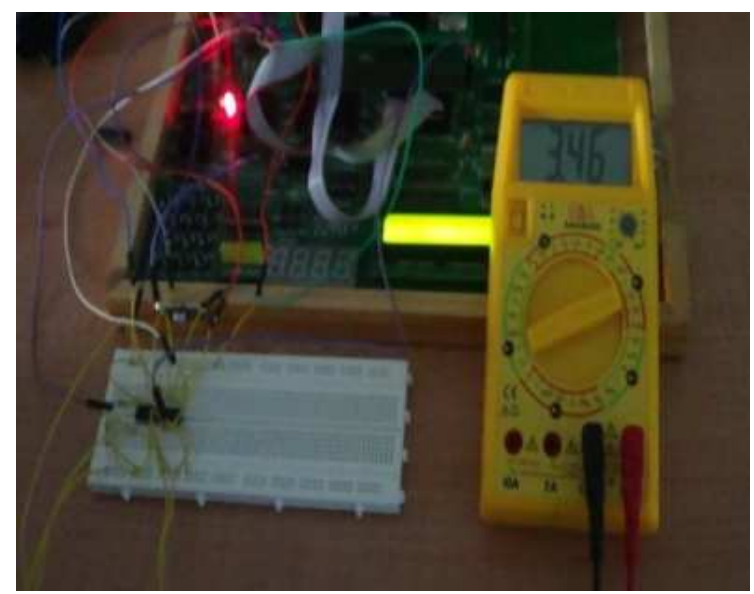

Fig. 14. Photograph of $A D C$ value display on $L C D$

\subsection{Ex.6. DC Motor Interfacing}

DC motor control is an important aspect in industry. Keeping this in view point, a DC motor is interfaced via a power amplifier ICL293 [12]. The direction of rotation is determined by the IC MOC70P2 [13]. Software is developed to control the rotational direction of DC motor and the speed of rotation using opto-sensor. The circuit assembled as shown in figure 15.

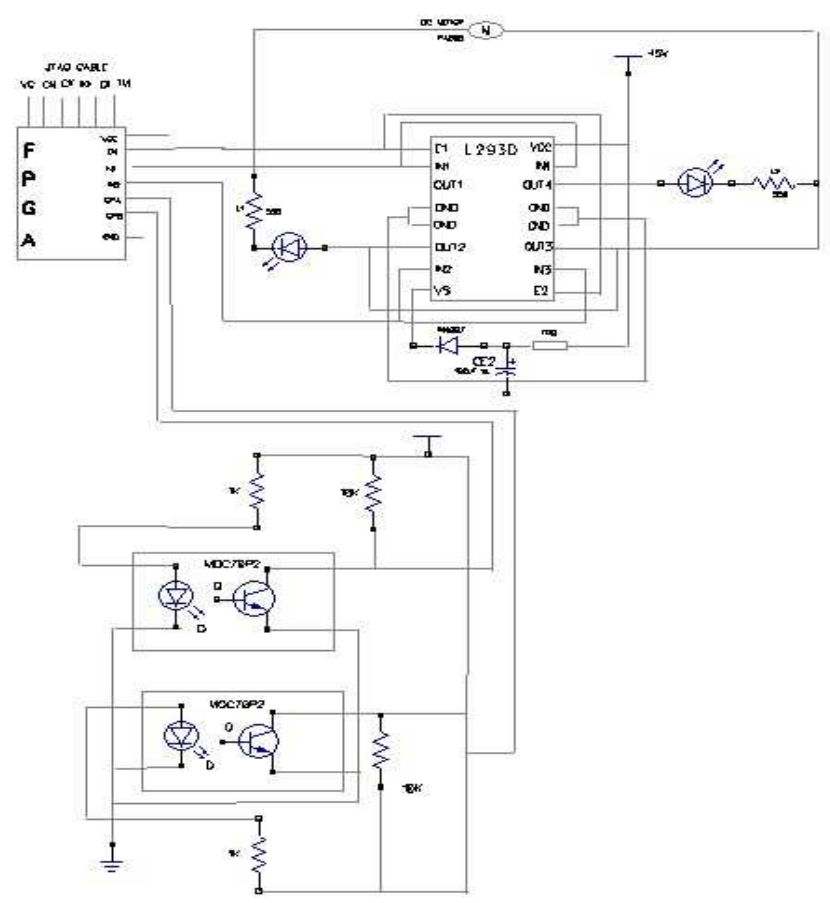

Fig. 15. Schematic diagram of DC motor interfacing 
The corresponding algorithm is given below:

1. Start

2. Initialize the Header 1 as output port

3. Initialize the Header 2 as input port

4. Measure speed and direction of dc motor and display continuously.

The photograph of the DC Motor interfacing experiment is shown in figure 16.

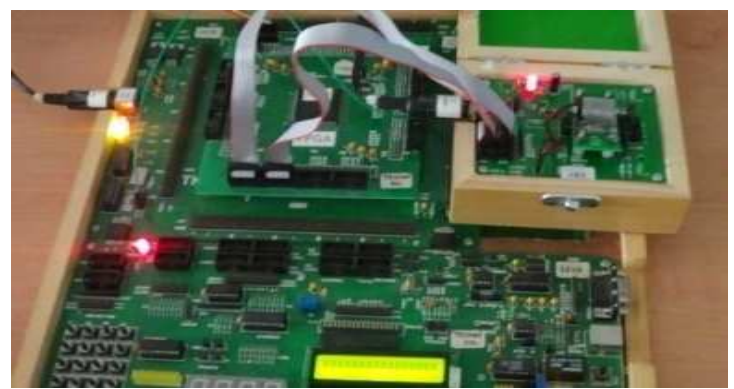

Fig. 16. Photograph of DC motor interfacing

\subsection{Ex. 7. Heart Beat Measurement System}

As a project-based experiment, we have designed and developed a heart beat measuring circuit. The FPGA based is interfaced with the heart beat sensor system [14]. The basic principle that underlies the measurement is the pulsating blood flow through artery of the finger tip. The schematic circuit diagram of heart beat measurement system is shown in figure 17.

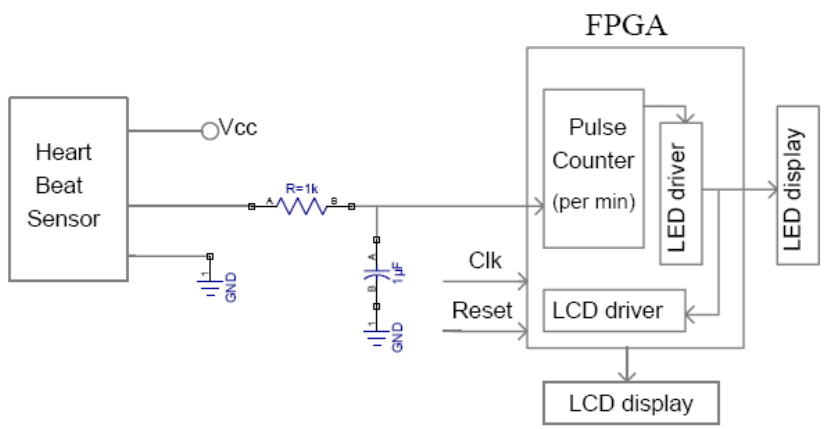

Fig. 17. Schematic diagram of Heart beat measurement system

Program for Heart Beat Measurement System is developed using below Algorithm:

1. Start

2. Initialize the pulse counter

3. Initialize the LCD communication

4. Display Heart Beat Pulses per minute on LCD

5. Display the heart beat pulses in the form of LED

The photograph of heartbeat measurement system is shown in figure 18.

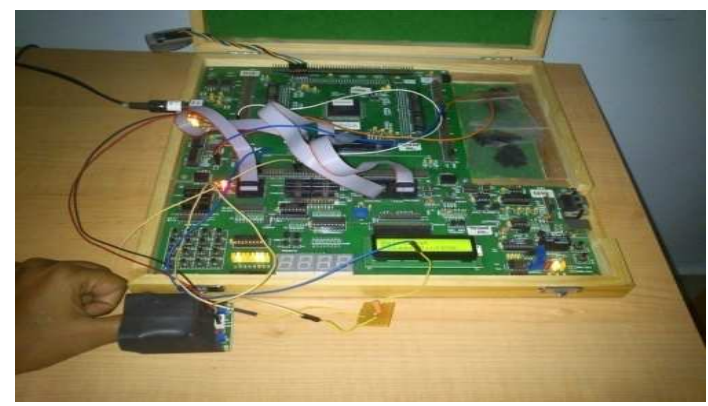

Fig. 18. Photograph of Heart beat measurement system

\section{Conclusion}

A series of experiments useful for Under-graduate ECE/CSE laboratory designed and developed in author's laboratory is explained in this paper. These experiments can be introduced in a laboratory course. The hands on experience a student get by performing these experiments definitely give a confidence to think and develop a complex FPGA-based interfacing which is very much need in the present scenario of hardware revolution.

\section{Acknowledgement}

C. Swapna is thankful to University Grants Commission (U.G.C.), New Delhi, for the sanction of Junior Research Fellowship (B.S.R.).

\section{References}

[1] Aruna Kommu, Raghavendra Rao Kanchi, "Design and development of a project-based embedded system laboratory using LPC1768", American Journal of Embedded Systems and Applications, Vol.1, No.2, November 2013. pp 46-53.

[2] John Bowles and Gang Quan, “An FPGA - Based Embedded System Design Laboratory for the Undergraduate Computer Engineering Curriculum", American society for Engineering Education, AC2009-761.

[3] www.xilinx.com/spartan3LC.pdf.

[4] Jayaram Bhaskar (1998): A "VHDL Primer", 3 rd Edition, PTR Prentice Hall.

[5] Applied Digital Microsystems (ADM) Pt, Ltd., Mumbai.

[6] Spartan - 3FPGA family: Complete datasheet DS099, 2009. http://www.Xilinx.com/support/documentation/data sheets/ds321.pdf.

[7] ADM TKB 208 user Manual - XC3SXX D-board, Applied Digital Microsystems, Mumbai, http://www.admlindia.com.

[8] JTAG Interface: simple introduction, www.amontec.com/amt-ann004.pdf.

[9] ISE 9.1i $\quad$ Quick Start http://www.xilinx.com/itp/Xilinx/books/docs/qst/ast.pdf.

[10] http://pdf/.alldatasheet.com/datasheet-pdf/view/111499/HIT $\mathrm{ACHI} / \mathrm{HD} 447$ 
[11] www.ti.com/lit/ds/symlink/adc0804-n.pdf

[12] www.ti.com/lit/ds/symlink/1293d.pdf

[13] http://datasheet.octopart.com/MOC70P2-Fairchild-datasheet $-8378515 . p d f$
[14] Swapna. C, Vijay Kumar. J, Raghavendra Rao. K, Ramanjappa.T,"FPGA Based Heart Beat Measurement System", International Conference on Information Technology, Electronics and communications (ICITEC), July 14 - 15, 2012, Hyderabad, India. 